\title{
Quantitative Ultrasound Texture Analysis of Fetal Lung Versus Fetal Pulmonary Artery Doppler as a Predictor of Neonatal Respiratory Distress Syndrome (RDS)
}

\author{
Mohamed Kamaleldin Abdelhamid ${ }^{1}$, Hosny Said Abdel Ghani ${ }^{1}$, Osama Abdel Wadood Khalil ${ }^{1}$, \\ Saad El-Gelany ${ }^{2}$, Nasr Mohamed Osman, ${ }^{1, *}$ \\ ${ }^{1}$ Radiology Department, Faculty of Medicine, Minya University, Minya, Egypt \\ ${ }^{2}$ Gyna and Obstetric Department, Faculty of Medicine, Minya University, Minya, Egypt
}

Email address:

Mohamed.kamal@gt.com (M. K. Abdelhamid), hosnyabdelghani@hotmail.com (H. S. A. Ghani), ascannet@yahoo.com (O. A. W. Khalil), saad.elgelany@yahoo.co.uk (S. El-Gelany),nasrosman_7@yahoo.com (N. M. Osman)

${ }^{*}$ Corresponding author

To cite this article:

Mohamed Kamaleldin Abdelhamid, Hosny Said Abdel Ghani, Osama Abdel Wadood Khalil, Saad El-Gelany, Nasr Mohamed Osman. Quantitative Ultrasound Texture Analysis of Fetal Lung Versus Fetal Pulmonary Artery Doppler as a Predictor of Neonatal Respiratory Distress Syndrome (RDS). International Journal of Medical Imaging. Vol. 8, No. 2, 2020, pp. 23-34. doi: 10.11648/j.ijmi.20200802.12

Received: April 23, 2020; Accepted: May 13, 2020; Published: June 3, 2020

\begin{abstract}
Purpose: To evaluate the role of fetal pulmonary artery Doppler indices and quantitative ultrasound texture analysis of the fetal lung (quantus FLM) in prediction of neonatal respiratory distress syndrome. Method: This Observational prospective cross sectional study included 40 pregnant women between 28 and 40 weeks gestation. The diagnostic accuracy of MPA Doppler measurements (pulsatility index (PI), resistance index (RI) and acceleration time/ejection time (AT/ET) for diagnosis of neonatal RDS was tested by comparing the Doppler findings with the clinical outcome. Results: Of the 40 eligible fetuses, 9 (22\%) developed neonatal RDS There was a significant correlation between the AT/ET and the development of the $\mathrm{RDS}$ as the AT/ET was significantly lower in the RDS +ve group (mean 0.27 ) in comparison to the RDS-ve group (mean 0.34 ) (P 0.001). While both PI and RI showed no statistically significant difference in between the two groups. A cutoff value of 0.3 for AT/ET predicted the development of RDS (sensitivity: 77.78\%, specificity: 83.87\%). Quantus FLM predicted the development of neonatal RDS with high sensitivity, specificity, positive predictive value, negative predictive value and accuracy $(88.98 \%, 90.32 \%, 72.7 \%, 96.6 \%$ and $90 \%$ respectively). The area under curve was 0.896 . Conclusion: Neonatal RDS can be predicted using the MPA AT/ET and quantitative ultrasound texture analysis of the fetal lung (quantus FLM) with high sensitivity and specificity.
\end{abstract}

Keywords: Fetal Lung Maturity, Neonatal Respiratory Distress Syndrome, Color Doppler Fetal Pulmonary AT/ET, Quantus FLM

\section{Introduction}

Approximately $7.3 \%$ of all live births in Egypt are premature, meaning birth before 37 weeks of gestation [1]. Of the many complications of prematurity, lung diseases, remain the most common cause of neonatal morbidity $[2,3]$. Respiratory distress syndrome (RDS) of the newborn, also called hyaline membrane disease which is the most common cause of respiratory distress in premature infants, correlating with structural and functional lung immaturity and it is the commonest cause of neonatal mortality [4]. In those who are born prematurely, the obstetrician has to balance the benefits of a preterm birth on both mother and child against the obvious risks of preterm births for the neonates. So, detecting those fetuses at risk for RDS by accurate evaluation of fetal lung maturity is of utmost importance for determining the optimal time for pregnancy termination, especially in high risk pregnancies.

A number of biochemical tests have been developed to predict the risk of RDS. These tests, such as, the lecithin/sphingomyelin (L/S) ratio require amniocentesis. However, amniocentesis is an invasive procedure and is associated with a small but real risk to the pregnancy, including 
preterm premature rupture of membranes, preterm labor, placental abruption, fetomaternal hemorrhage, fetal injury, and rarely fetal or even maternal death [5]. Noninvasive methods using ultrasound to assess fetal lung maturity have long been proposed such as measurements of lung volumes, gestational age, epiphysis centers, placental grading, and estimated fetal weight, but none has been successful to this date [6-9]. So this prompted the physicians to try to investigate other noninvasive sonographic techniques to evaluate lung maturity.

\section{Patients and Methods}

\subsection{Study Population}

The study was reviewed and approved by the local institutional ethics and review committee of our University Hospital and was conducted in accordance with the Helsinki Declaration. Our study included 40 patients that delivered at Maternity and Children University Hospital in the period from May 2018 till December 2018 with the following inclusion and exclusion criteria Inclusion criteria

1. Gestational age between $28-40 \mathrm{~W}$.

2. Ultrasonography done within 48 hours of delivery.

3. Singleton pregnancy.

Exclusion Criteria:

Pregnant females less than 28 weeks gestational age or those more than 40 weeks, multiple gestations, uncertain gestational age, corticosteroid administration between ultrasound examination, delivery and congenitally malformed fetus.

\subsection{Study Design}

Observational prospective cross sectional study

\subsection{Plan of Study}

This is summarized in Figure 1.

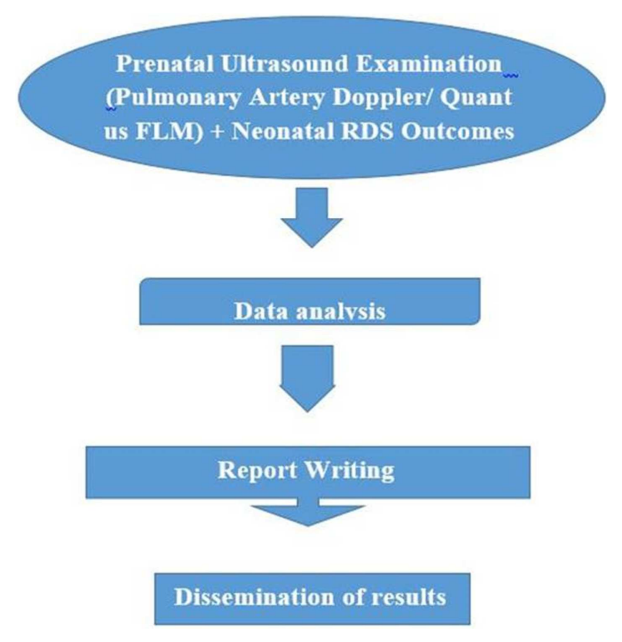

Figure 1. Plan of the study.

The study subjects were chosen from among cases delivering in Minia Maternity \& Children University Hospital, Egypt from the May 2018 to December 2018.

\subsection{Sonographic Antepartum Assessment}

Pregnant women were scanned in supine position. Routine prenatal sonographic examination includes fetal biometry (BPD, FL and HC) which were used to estimate the fetal gestational age and the expected fetal weight and to rule out intrauterine growth retardation or macrosomia. Amniotic fluid index was measured as well. An axial section of the fetal thorax at the level of the 4-chamber cardiac view was magnified by adjusting the depth, but not the zoom, until the thorax occupies about most of the screen, avoiding obvious acoustic shadows from the fetal ribs or spine (Figure 2). Images were acquired without any type of post processing manipulation such as Allipure, smoothing, color Doppler, or any calipers or pointers. By the use of tissue harmonic imaging and adjustment of image settings; such as gain, frequency, and time-gain obtain optimal images. The acquired images were submitted in DICOM format to the Quantus FLM online software for analysis and report generation (Figure 3). Quantus FLM is a novel computerized method based on texture analysis and machine learning algorithms that was developed using more than 13000 non-clinical images and 900 fetal lung images, trained to predict neonatal respiratory morbidity risk on fetal lung ultrasound images based on images textural features that are invisible to human eyes. It is composed of two modules, a texture feature extractor and a classifier. Features extracted from the acquired ultrasound image of the axial section of the fetal thorax at the level of the four-chamber cardiac view were used, by the classifier, to assess the risk of respiratory morbidity [10].

The fetal main pulmonary artery (MPA) was then visualized by slightly translating the transducer superiorly to obtain the three vessel view (3VV) (Figure 4). The pulsed Doppler sample gate is placed at the middle of the fetal MPA (between the pulmonary valves and the pulmonary artery bifurcation). After enlarging the image as much as possible, the sample gate was adjusted to $3 \mathrm{~mm}$.

Fetal MPA Doppler waveforms produce a specific "spike and dome" pattern, and a small notch at the end of systole (Figure 5a). This specific shape of MPA waveform is important to differentiate from ductus arteriosus waveform which is rounded, full and triangular shaped. After the optimal fetal MPA waveform were obtained, relevant

Doppler velocity variables are measured three times using manual trace, and measurements are averaged. The Doppler variables include acceleration time (AT; the time interval from the foot to the top of PSV), ejection time (ET; from the beginning to the end of ventricular systole), From these measurements, the AT/ET ratio was calculated (Figure 5b).

Other Doppler parameters were measured using automatic trace such as the maximum blood flow velocity reached during systole (PSV), end-diastolic maximum velocity (EDV), pulsatility index (PI), and resistance index (RI).

Post-partum assessment of each neonate included

1. Birth weight

2. APGAR 1,5 minutes

3. Occurrence of RDS 
4. X-ray chest if clinically indicated

5. The need of NICU admission

6. Need of a respiratory support (nasal CPAP, mechanical ventilation, endotracheal intubation)

7. Neonates with the following were excluded from the analysis:

Respiratory distress due to causes other than RDS and conditions such as: neonatal sepsis, hemodynamic failure, symptomatic anemia and meconium aspiration, as these conditions could directly predispose or lead to NRM, irrespective of lung maturity.

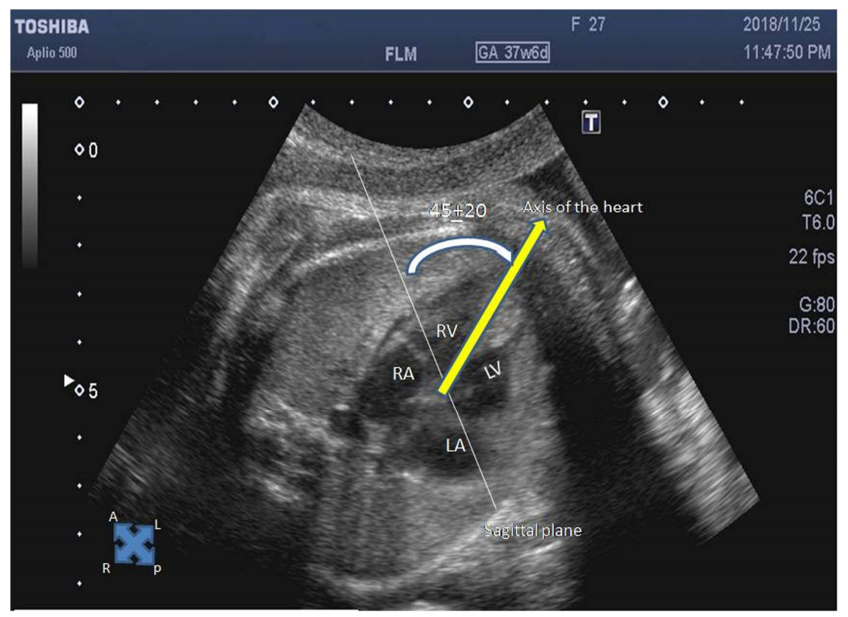

Figure 2. The 4-chamber view of the Heart of one of the studied fetuses.

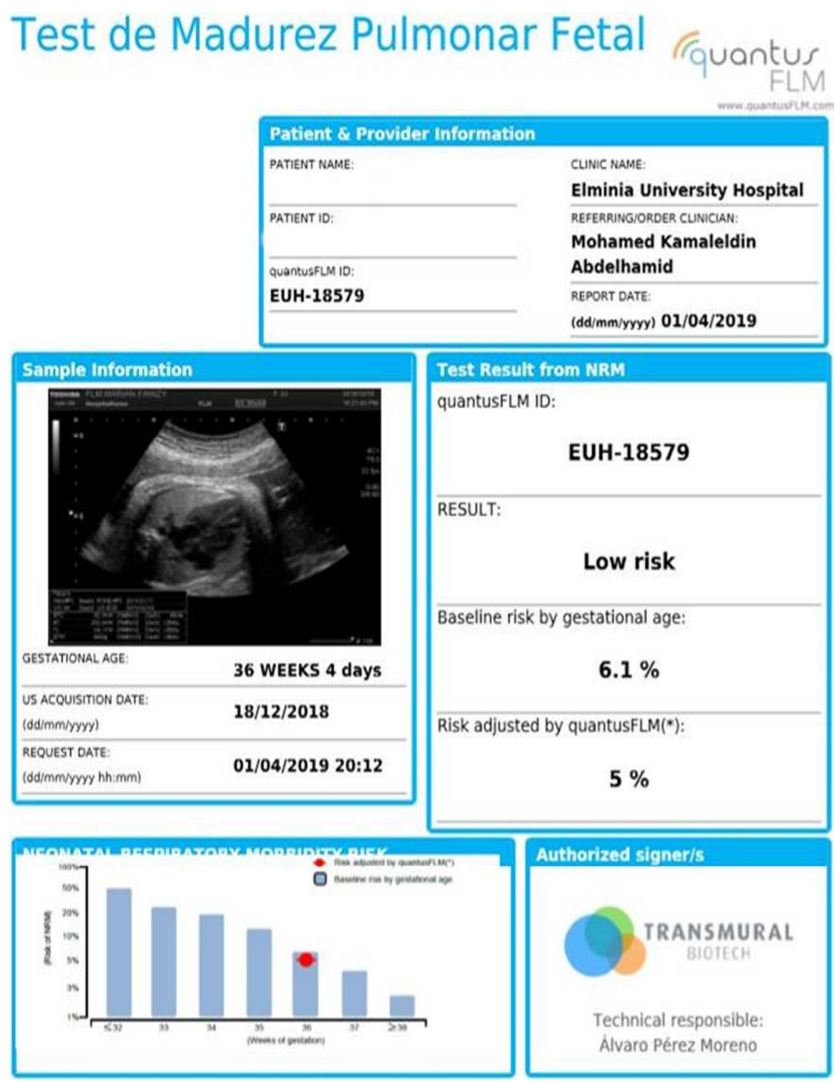

Figure 3. Quantus FLM report of one case in our study.
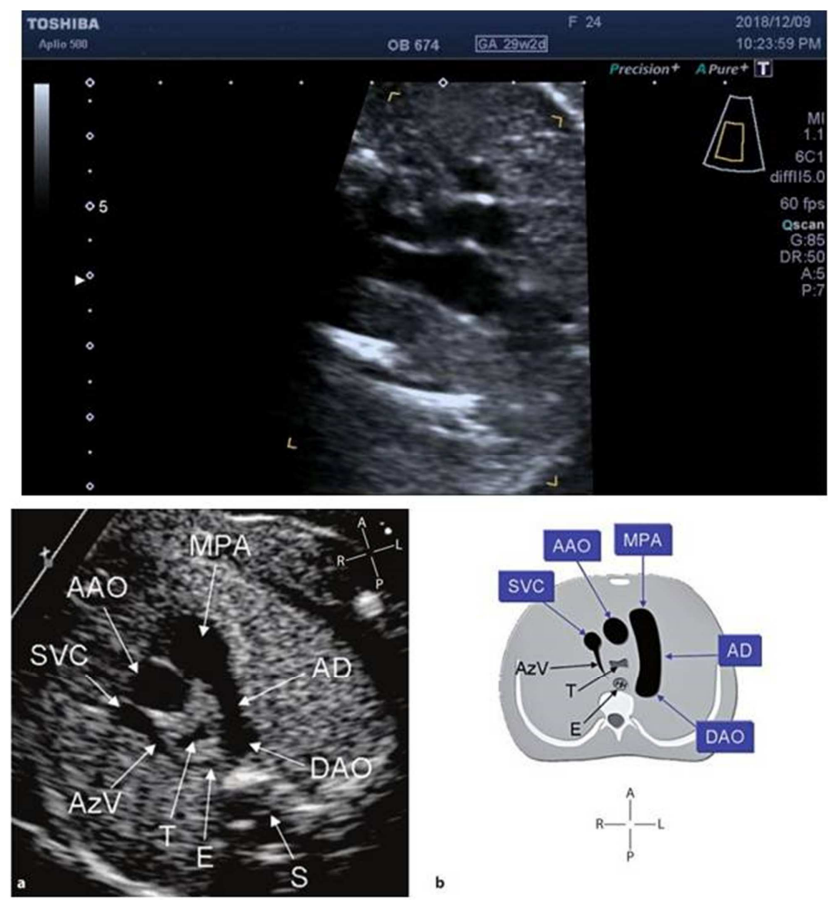

Figure 4. The three vessels view
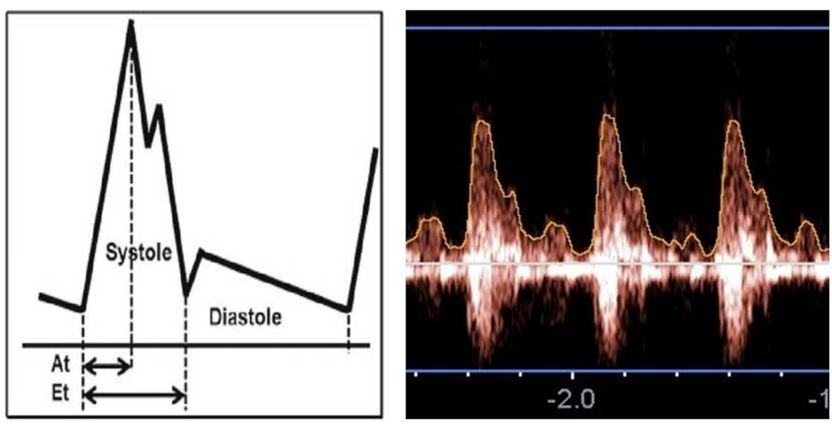

Figure 5. Acceleration and ejection time. Spike and Dome Pattern ${ }^{[140]}$.

\subsection{Diagnosis of Neonatal Respiratory Distress Syndrome}

Neonatal RDS was diagnosed by neonatologist, blinded to fetal MPA Doppler or Quantus FLM results if at least 2 of 3 from these criteria were present:

1) Clinically: Evidence of respiratory failure (tachypnea, retraction and, or nasal fairing) shortly after birth and increased oxygen requirement (fractional concentration of inspired oxygen $>0 / 4$ ) for more than 24 hours.

2) Radiographic: Evidence of hyaline membrane disease such as: reticulo-nodular pattern, air bronchogram and ground glass appearance in the absence of other causes of respiratory disease.

3) Response to exogenous pulmonary surfactant.

In the current study, the diagnosis of neonatal RDS was based on clinical and radiological assessment.

\subsection{Equipment}

All sonographic examinations were done by one examiner using Aplio ${ }^{\text {TM }} 500$ ultrasound (Toshiba Medical Systems Co. Ltd, Tamara, Japan) equipped with a 3.5 to $5.0 \mathrm{MHz}$ convex transducer. 


\subsection{Outcome Measures}

Recording the clinical outcome of the development of neonatal respiratory distress syndrome (RDS) and comparing it with antepartum sonographic examinations. Details of pregnancy outcome for the participants were obtained from the maternity case records. The clinical outcomes were compared against Doppler and quantus FLM estimates. Our Results will inform future, appropriately powered, studies aimed at elucidating any differences in the subsets or categories of women with pregnancies complicated with RDS, classified by etiological associations. These further studies are likely to require larger sample sizes over a longer period.

\subsection{Data Statistical Analysis}

Statistical advice regarding study design was obtained from the Statistical Services Unit (SSU) of the University. The data were coded, entered and processed on an IBM-PC compatible computer using SPSS (version 20). The level P $<0.05$ was considered the cut-off value for significance. Graphics were done by Microsoft Excel 2016.

The following tests were used in statistical analysis:

i. Description of quantitative variables as mean, SD and range

ii. Description of qualitative variables as number and percentage

iii. Independent samples $\mathrm{T}$ test for parametric quantitative data between the two groups

iv. Mann Whitney test for Non-parametric quantitative data between the two groups

v. Fisher exact test for qualitative data between the two groups

vi. The diagnostic accuracy of Doppler parameters and Quantus FLM was assessed using receiver operating characteristic (ROC) curve, which plot the sensitivity (true-positive rate) to the specificity (false-positive rate)

\subsection{Software Used}

SPSS (Statistical package for social sciences) version 20 was used during analysis. Endnote X7: reference manager, volume license copy (Bld 2114). Microsoft Excel 2016 was used for graphics. MedCalc statistical software program version 12.1.4.

\subsection{Health Safety Regulation and Work Schedule}

The biological specimens, materials and reagents were handled according to health and safety regulations, settled by our University hospitals. Work Schedule or Timeline: Summarized at Table 1.

Table 1. The time table of the study.

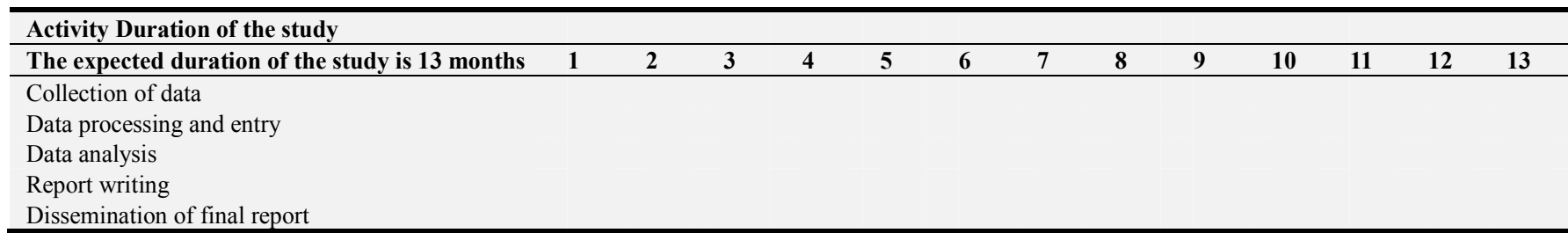

\section{Results}

Total of (65) patients were examined (Table 2). Twentyfive cases were excluded from the study: 14 patients of them gave birth more than 48 hours after the ultrasound examination, 8 cases had respiratory distress due to causes, 2 cases were discarded for missed neonatal data and one case for suboptimal ultrasound data. Finally, (40) fetuses entered the study, among which 9 were diagnosed as RDS (+ve) and 31 were RDS (-ve). Figure 6 represents the flow chart of the cases enrolled in the study.

There were no statistically significant differences between the neonates with RDS and those without RDS in terms of maternal age and parity however, the mean gestational age was $32.7+/-2.9 \mathrm{~W}$ in those neonates who developed RDS (RDS +ve group), compared to $37.6+/-1.5 \mathrm{~W}$ in those neonates who did not develop RDS (RDS -ve group). The difference between the two group was statistically significant (P value $<0.001$ ) (Table 2 ).

There were statistically significant differences between the two groups in terms of gestational age, amniotic fluid index, neonatal weight, 5min APGAR score and the need for NICU admission. The RDS +ve group had significantly lower gestational age, measured by ultrasound, (mean 32.7 w), AFI (median 7.6) and weight (median $2159.6 \mathrm{gm}$ ) in comparison to the RDS-ve group. All RDS+ve neonates were admitted to NICU (100\%) while in the RDS-ve group just 3 cases $(9.7 \%)$ were admitted to NICU. 5 minutes Apgar score was significantly lower in neonate who subsequently developed RDS. Fetal gender and $1 \mathrm{~min}$ APGAR score showed no statistically significant relation (Table 3). Table 6 shows that there was a statistically significant difference $(\mathrm{P}$ value $<0.001)$ between the two groups. 8 neonates $(88.9 \%)$ whom were classified as a high risk according to the Quantus FLM results, developed RDS. Another 3 neonates $(9.7 \%)$ were also classified as high risk of developing RDS without actually developing it. In total, 29 neonates were classified as a low risk for RDS development. 28 cases $(90.3 \%)$ did not develop RDS, while only 1 case developed RDS (11.1\%) (Table 4).

These results point to the fact that a high risk quantus FLM is a good predictor of later development of neonatal RDS and vice versa. There was a significant correlation between the $\mathrm{AT} / \mathrm{ET}$ and the development of the RDS as the AT/ET was significantly lower in the $\mathrm{RDS}+\mathrm{ve}$ group (mean 0.27 ) in 
comparison to the RDS-ve group (mean 0.34). While both PI and RI showed no statistically significant difference in between the two groups.

According to Table 4 quantus FLM predicted the development of neonatal RDS with high sensitivity, specificity, positive predictive value, negative predictive value and accuracy $(88.98 \%, 90.32 \%, 72.7 \%, 96.6 \%$ and $90 \%$ respectively). The area under curve was 0.896 . (Figures $7,8)$

According to Table 5 and Figure 9 AT/ ET mean in MPA Doppler velocimetry is significantly lower in fetuses which subsequently developed $\operatorname{RDS}(0.27+/-0.05)$ than those without RDS $(0.34+/-0.05)$, (P-Value $<0.001)$. It means that AT / ET ratio is positively correlated with RDS outcome Considering AT/ET cutoff point as 0.3 , RDS can be diagnosed with high sensitivity, specificity, positive predictive value, negative predictive value and accuracy (77.78\%, 83.87\%, 58.3\%, 92.9\% and $82.5 \%$ respectively). It means that ratio $>0.3$ demonstrates mature fetal lung. The area under curve was 0.844 . According to Table 6 there was no significant statistic difference between quantus FLM and AT/ET ROC curves (Figures 10, 11, Table 6).

Table 2. Maternal Data.

\begin{tabular}{|c|c|c|c|c|}
\hline & & RDS -Ve & RDS + Ve & D noln \\
\hline & & $\mathbf{N}=31$ & $\mathbf{N}=\mathbf{9}$ & Pvalue \\
\hline \multirow{2}{*}{ Age } & Range & $(19-38)$ & $(17-42)$ & \multirow{2}{*}{0.551} \\
\hline & Mean $\pm S D$ & $26.1 \pm 5.5$ & $27.9 \pm 8.4$ & \\
\hline \multirow{2}{*}{ Parity } & $P G$ & $7(22.6 \%)$ & $3(33.3 \%)$ & \multirow{2}{*}{0.327} \\
\hline & $M G$ & $24(77.4 \%)$ & $6(66.7 \%)$ & \\
\hline \multirow{2}{*}{ GA (LMP) } & Range & $(31-39)$ & $(28.7-37.3)$ & \multirow{2}{*}{$<0.001 *$} \\
\hline & $M e a n \pm S D$ & $37.6 \pm 1.5$ & $32.7 \pm 2.9$ & \\
\hline
\end{tabular}

Independent samples $\mathrm{T}$ test for parametric quantitative data between the two groups

Mann Whitney test for Non-parametric quantitative data between the two groups

Fisher exact test for qualitative data between the two groups

*: Significant difference at $\mathrm{P}$ value $<0.05$

Table 3. Fetal and neonatal data.

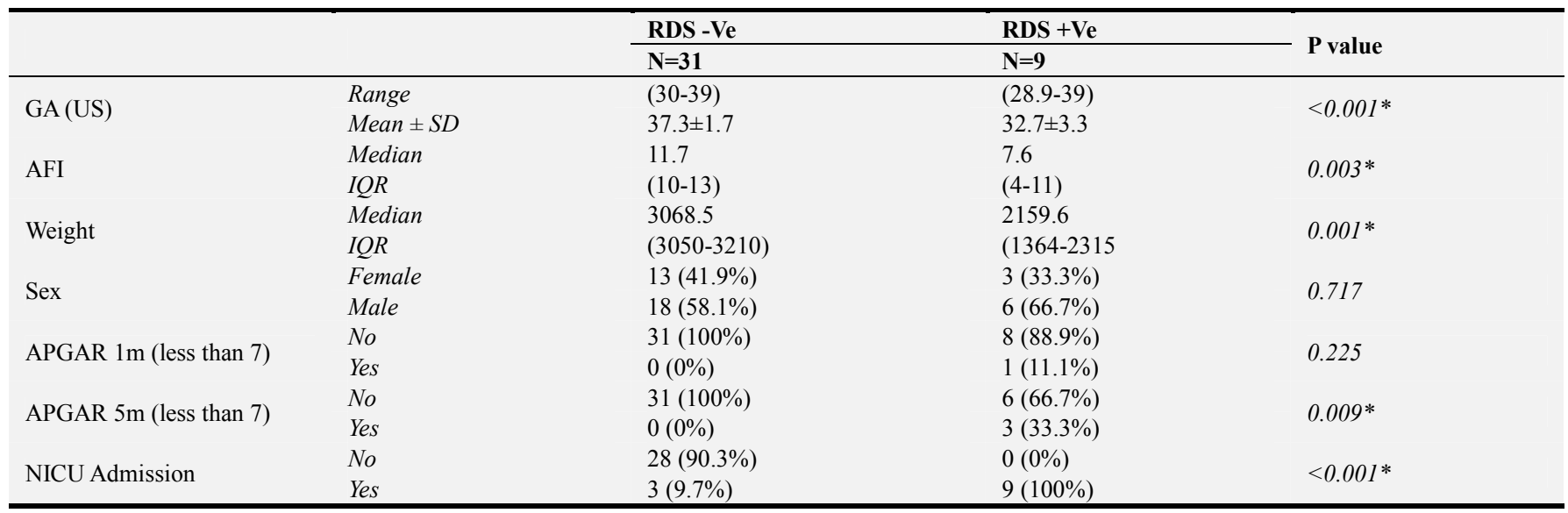

Independent samples T test for parametric quantitative data between the two groups

Mann Whitney test for Non-parametric quantitative data between the two groups

Fisher exact test for qualitative data between the two groups

*: Significant difference at $\mathrm{P}$ value $<0.05$

Table 4. QuantusFLM results.

\begin{tabular}{llll}
\hline & & RDS - Ve & No \\
\cline { 2 - 3 } & $\mathbf{N}=\mathbf{3 1}$ & $1(11.1 \%)$ \\
\hline \multirow{2}{*}{ Quantus FLM } & Low risk & $28(90.3 \%)$ & $8(88.9 \%)$ \\
\hline
\end{tabular}

Fisher exact test for qualitative data between the two groups

*: Significant difference at $\mathrm{P}$ value $<0.05$

Table 5. MPA Doppler indices.

\begin{tabular}{llll}
\hline & & RDS - Ve & RDS +Ve \\
\cline { 2 - 3 } & & N=31 & N=9 \\
\hline \multirow{2}{*}{ RI } & Range & $(0.55-0.99)$ & $(0.57-0.93)$ \\
& Mean $\pm S D$ & $0.82 \pm 0.11$ & $0.77 \pm 0.13$ \\
\hline
\end{tabular}




\begin{tabular}{|c|c|c|c|c|}
\hline & & RDS -Ve & RDS +Ve & \\
\hline & & $\mathbf{N}=\mathbf{3 1}$ & $\mathbf{N}=9$ & P value \\
\hline PI & $\begin{array}{l}\text { Range } \\
\text { Mean } \pm S D\end{array}$ & $\begin{array}{l}(1.67-3.54) \\
2.43 \pm 0.48\end{array}$ & $\begin{array}{l}(1.5-3.1) \\
2.24 \pm 0.56\end{array}$ & 0.317 \\
\hline $\mathrm{AT} / \mathrm{ET}$ & $\begin{array}{l}\text { Range } \\
\text { Mean } \pm S D\end{array}$ & $\begin{array}{l}(0.19-0.4) \\
0.34 \pm 0.05\end{array}$ & $\begin{array}{l}(0.2-0.35) \\
0.27 \pm 0.05\end{array}$ & $0.001 *$ \\
\hline
\end{tabular}

Independent samples $\mathrm{T}$ test for parametric quantitative data between the two groups

*: Significant difference at $\mathrm{P}$ value $<0.05$

Table 6. ROC curve analysis of AT/ET and Quantus FLM for prediction of RDS +Ve neonates.

\begin{tabular}{lll}
\hline & AT/ET & Quantus FLM \\
\hline Optimal cut off & $\leq 0.3$ & High Probability \\
AUC & 0.844 & 0.896 \\
$95 \%$ CI & $0.695-0.939$ & $0.758-0.970$ \\
P value & $<0.001 *$ & $<0.001 *$ \\
Sensitivity & $77.78 \%$ & $88.89 \%$ \\
Specificity & $83.87 \%$ & $90.32 \%$ \\
PPV & $58.3 \%$ & $72.7 \%$ \\
NPV & $92.9 \%$ & $96.6 \%$ \\
Accuracy & $82.5 \% \%$ & $90 \%$ \\
\hline
\end{tabular}

AUC: Area Under Curve

CI: Confidence Interval

PPV: Positive predictive value

NPV: Negative Predictive value

*: Significant level at $\mathrm{P}$ value $<0.05$

Table 7. Difference between FLM vs AT/ET ROC curves.

\begin{tabular}{ll}
\hline Difference between areas & 0.0520 \\
$95 \%$ CI & -0.128 to 0.232 \\
$Z$ statistic & 0.565 \\
P value & 0.5723 \\
\hline
\end{tabular}

Z statistics

Significant level at $\mathrm{P}$ value $<0.05$

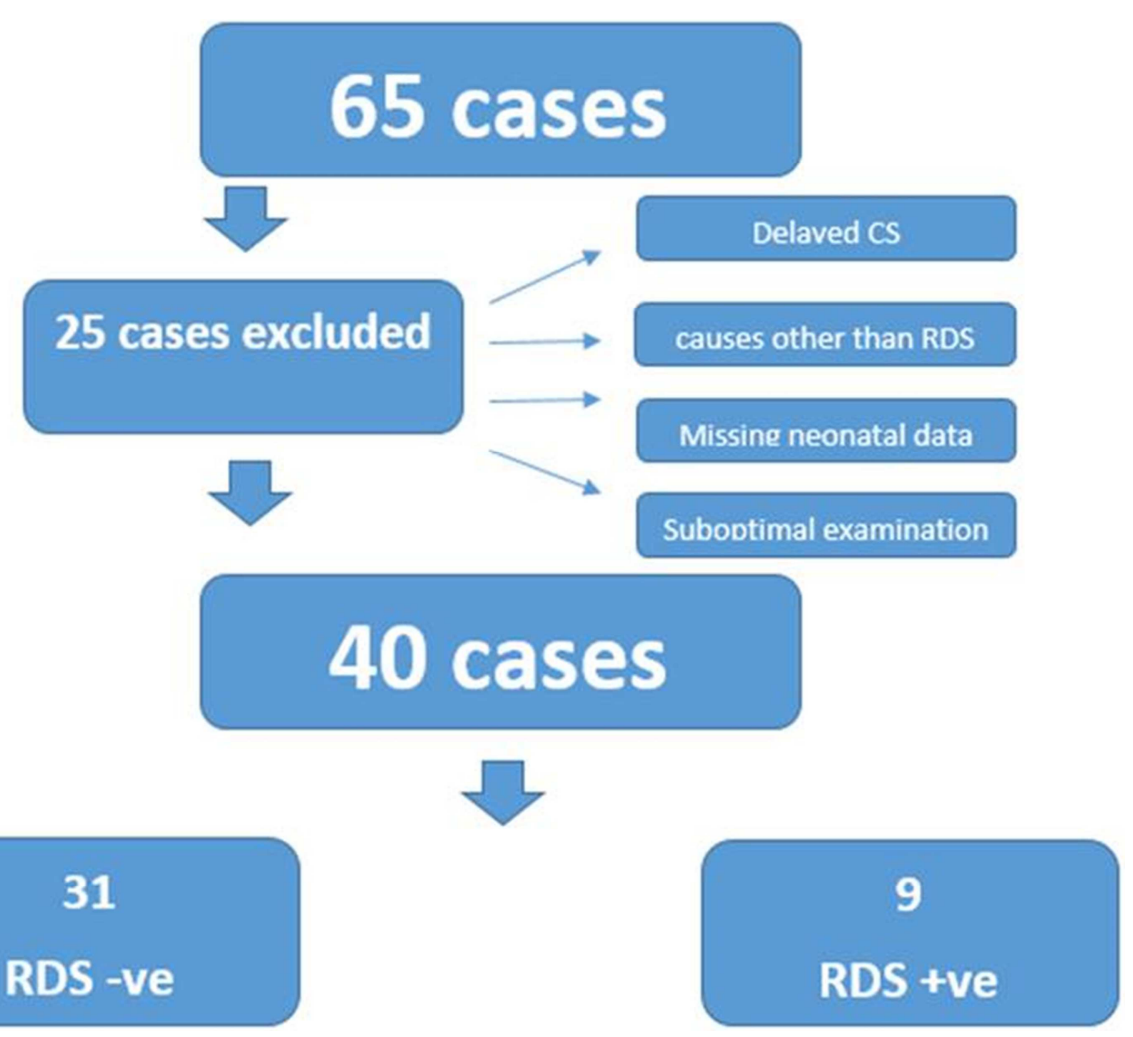

Figure 6. Flowchart of the study. 

Artery Doppler as a Predictor of Neonatal Respiratory Distress Syndrome (RDS)

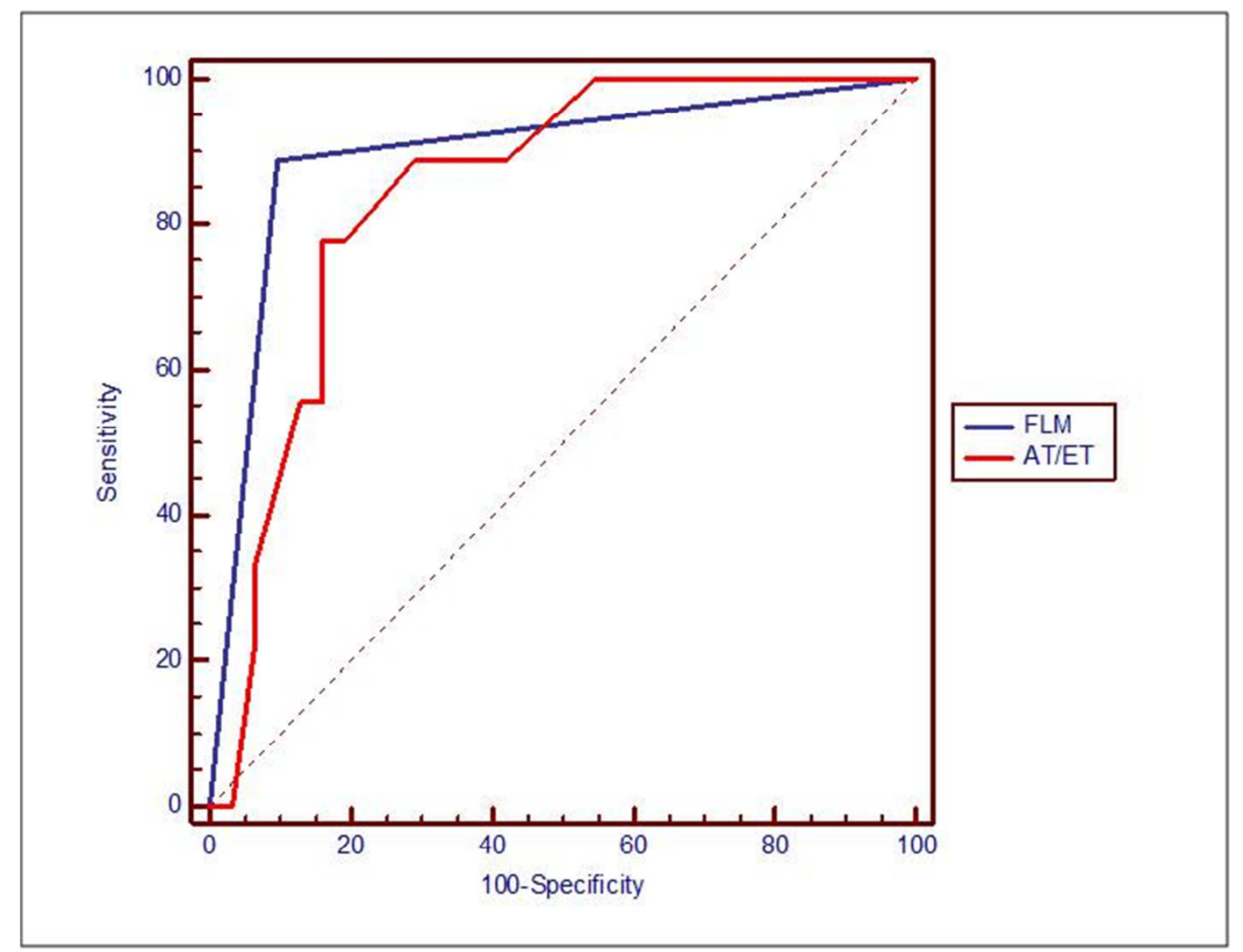

Figure 7. ROC curve.

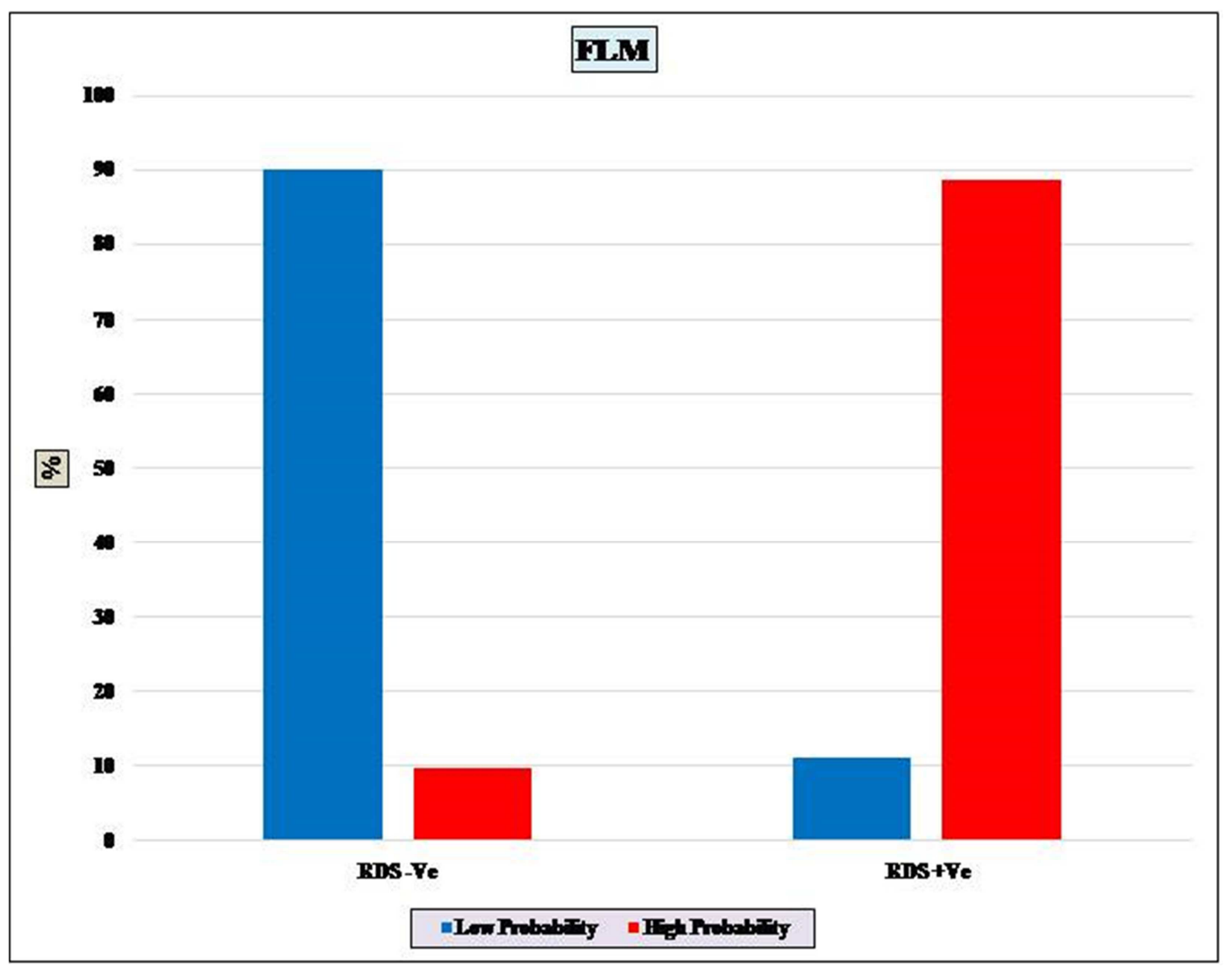

Figure 8. Quantus FLM in the two groups. 


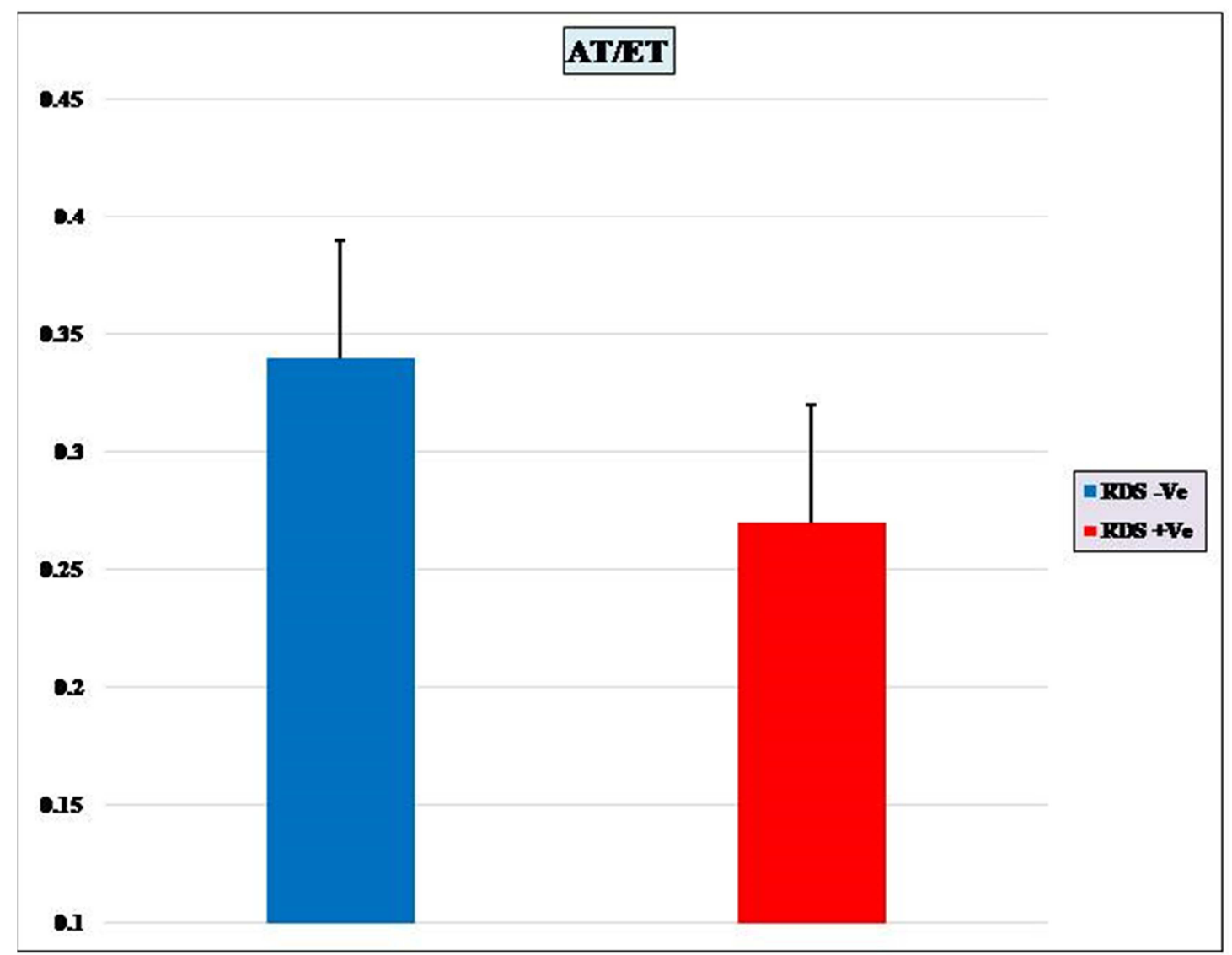

Figure 9. AT/ET in the two groups.

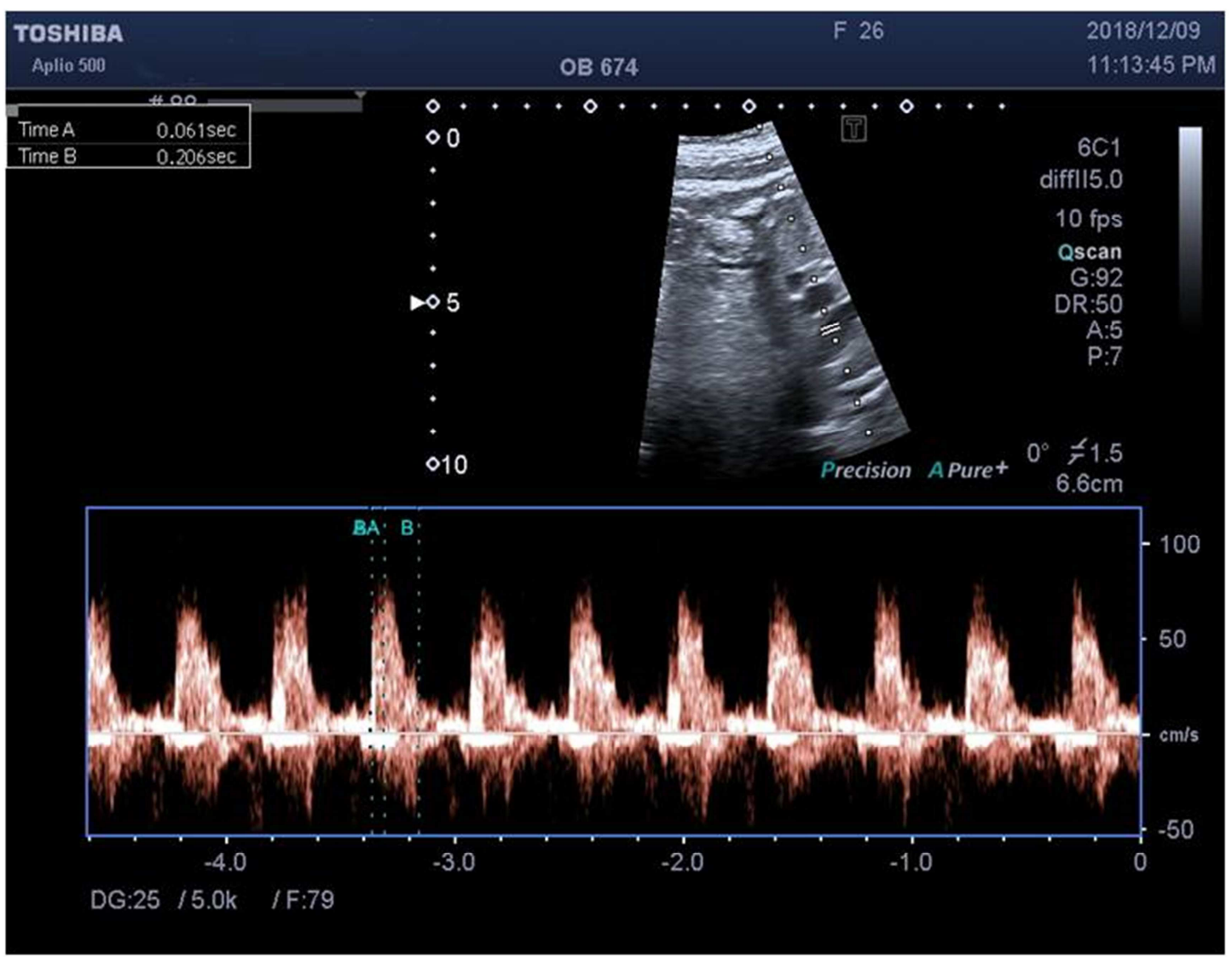

Figure 10. $A T=0.061, E T=0.206 A T / E T=0.296$. 


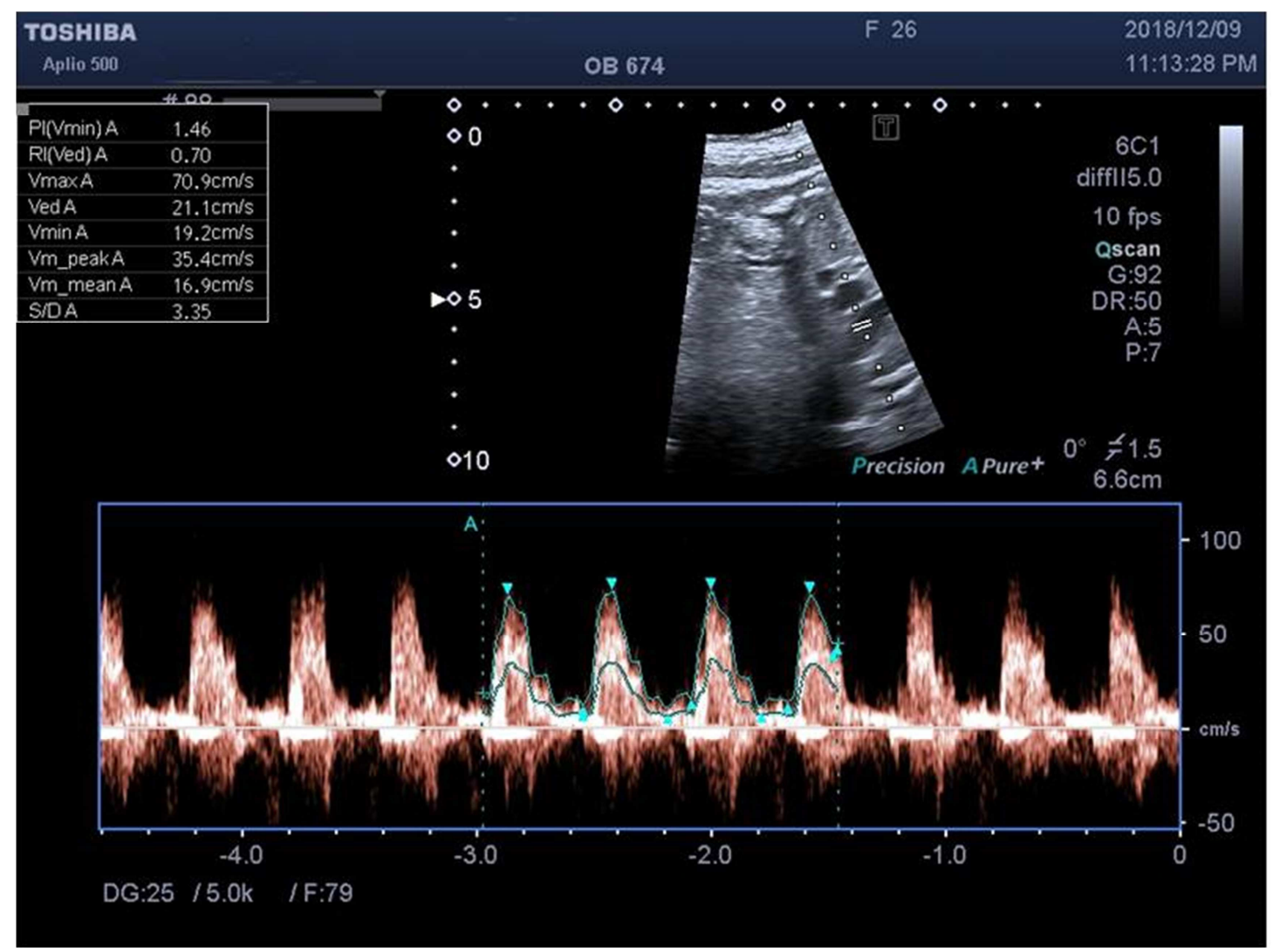

Figure 11. $R I=0.7, P I=1.46$.

\section{Discussion}

Respiratory distress syndrome (RDS) of the newborn is the most common cause of respiratory distress in premature infants [4]. This represents an imperative issue in our country as $7.3 \%$ of all live births in Egypt are premature [1]. The steady increase in the number of caesarian sections in our hospitals remains a major contributing factor to this issue, leading to an increase in fetal mortality and morbidity [1]. Proper evaluation of fetal lung maturity is of utmost importance for determining the optimal time for pregnancy termination to help decrease the incidence of RDS. However, all the available biochemical tests for lung maturity evaluation require amniocentesis which is an invasive procedure that carries major risks including preterm premature rupture of membranes, preterm labor, placental abruption, fetomaternal hemorrhage, fetal injury, and rarely fetal or even maternal death [5].

This prompted us to investigate two noninvasive sonographic techniques to evaluate fetal lung maturity, Quantitative ultrasound texture analysis of fetal lung and fetal pulmonary artery Doppler, comparing their value in the prediction of neonatal RDS. Our results showed that quantus FLM predicted the development of neonatal RDS with high sensitivity, specificity, positive predictive value, negative predictive value and accuracy $(88.98 \%, 90.32 \%, 72.7 \%, 96.6 \%$ and $90 \%$, respectively).

To the best of our knowledge this is the first report on using quantus FLM to evaluate fetal lung maturity in Egypt and North Africa; thus, we did not find any local previous studies for comparison and were obliged to compare our results with western reported studies. Our results were in agreement with Bonet-Carne et al. [10] and Palacio et al. [11], who concluded that quantus FLM provides a noninvasive tool that might help clinicians in the decision making process with an accuracy similar to that previously reported for other invasive tests with the advantage of being a noninvasive technique. In our study using quantus FLM, a higher amount of high-risk results was observed in neonates with low birth weight and low gestational age at delivery. A possible explanation is the fact that the incidence of RDS is inversely proportionate to the gestational age [12] or that the algorithm takes the gestational age at delivery under account in the analysis. If the latter is the case, the software needs to be adjusted for different gestational ages to ensure that gestational age at delivery does not work as a confounder as there might be a risk that the Quantus-FLM is determining whether a fetus is preterm or full-term rather than predicting the risk of neonatal RDS. In this study, we found rather difficult to obtain a longitudinal section in the fetal main pulmonary artery. Therefore, an accurate correction of the insonation angle could not be achieved; thus, we did not include pulmonary artery velocimetry parameters (PSV, EDV, $\mathrm{S} / \mathrm{D}$ ) in our results. This also may explain the wide variability of those parameters in previous studies of the fetal pulmonary artery Doppler [13,14, and 15]. We resorted to parameters that were not significantly affected by the angle 
of insonation; such as, acceleration time to ejection time ratio $(\mathrm{AT} / \mathrm{ET})$, pulsatility index (PI) and resistance index (RI). There was a significant correlation between the AT/ET and the development of the RDS as the AT/ET was significantly lower in the RDS +ve group (mean 0.27) in comparison to the RDS -ve group (mean 0.34), (P value 0.001). While both PI and RI showed no statistically significant difference in between the two groups, $P$ values 0.317 and 0.247 respectively.

An AT/ ET cut of point of 0.3 predicted the development of neonatal RDS with a high sensitivity, specificity, negative predictive value and accuracy $(77.78 \%, 83.87 \%, 92.9 \%$ and $82.5 \%$ respectively). Our results were in agreement with a study done earlier in the Obstetrics and Gynecology department in our hospital regarding the significant correlation between the AT/ET and the development of RDS. However, in contrast to our results, they also found a significant correlation between the RI and RDS development. This may be due to the different gestational age groups included in both studies [16]. Our results were also in agreement with Guan et al. [17], Moety et al. [13], Khanipouyani et al. [18] and Büke et al [19]. All of them had similar study designs (comparing the fetal main pulmonary artery Doppler with

Clinical outcome) and also Schenone et al study [20]. Who compared the AT/ET to albumin/surfactant ratio. This significant positive correlation between the AT/ET and fetal lung maturity may be explained as the elevated AT/ET represents a decrease in fetal pulmonary arterial pressure which is expected in a mature fetal lung [21]. However, our results contradicted Kim et al [14] and Mohamed et al. [22] who found that Pulmonary artery AT/ET ratio was inversely correlated to fetal lung maturity. The discordance may be due to a variation in study population or details of the ultrasound measurements and equipment. In this study the researcher was not able to spend much time at the delivery ward every day due to other duties, as radiology resident, in parallel. To be able to make good timings of ultrasound image acquisitions in relation to delivery, it has become evident during this study that it is of great importance that the researcher can indeed spend a lot of time at the delivery wards, being well informed by the physicians concerning the possible candidate's health and delivery status. The strategy in this study was to collect ultrasound images of the possible candidates that fulfilled the inclusion criteria when possible and then delete the images if delivery did not occur within 48 hours. The disadvantage of not being able to spend time at the delivery wards every day lead to missing to include a lot of neonates who eventually developed a respiratory morbidity. This disadvantage is something to take into account when developing similar studies in the future. Important to mention is the impossibility to predict whether a fetus will or will not develop RDS and also the difficulties in estimating whether or not a fetus will be delivered within 48 hours from the image acquisition. The inclusion criteria provided a very small time window in which the image could be obtained and this combined with the researcher's prerequisites made it even more difficult. This was reflected on our results as shown in the flow chart of our study (Figure 6), where 25 cases were excluded out of 65 cases, so only 40 cases were eligible for further analysis. Analysis of maternal data results (Table 2) showed that both maternal age and parity did not affect the development of RDS. Our study showed that both gestational age and fetal weight were significantly correlated with development of neonatal RDS as both were lower on those who eventually developed neonatal RDS ( $\mathrm{P}$ value $<0.001$ ). This is in agreement with the previous reports $[20,13,14,15,18]$, making gestational age and hence prematurity the most important factor in neonatal RDS development. Our results showed that fetal sex did not affect the occurrence of RDS; this is in contrast to the known male disadvantage in respiratory [23]. However, this can be attributed the low number of cases included in our study and we cannot predict the results if the study is repeated on a large scale. All RDS +ve neonate were admitted to NICU $(100 \%)$, while only 3 cases (9.7percent) in the RDS -ve group were admitted to NICU. This is expected as all who developed RDS were assumed to be admitted to NICU. One of the values of using these noninvasive techniques is guiding the clinical decisions concerning delivery planning and administering antenatal steroids. The clinician has to balance the benefits of a preterm birth on both mother and child against the obvious risks of preterm births for the neonate. Another theoretical possible way of using a highrisk result would be to use as an indication for antenatal steroids, to enhance fetal lung maturity and to estimate the deleterious effects of the antenatal steroids given. It is obvious from the results of our study that both quantus FLM and fetal pulmonary artery AT/ET are comparable in predicting fetal lung maturity and hence development of neonatal RDS.

The advantages of quantus FLM were the ease to obtain the required ultrasound images and the reproducibility of the results which eliminates the interobserver variations. However, it is a costly test especially in an economically disadvantaged country as ours and it also requires uploading the acquired images to online software before getting results. In contrast, Doppler is readily available on many ultrasound machines and the results can be obtained immediately. However, it is a rather difficult technique, heavily operator dependent and requires a great deal of experience. Therefore, there is a great deal of interobserver variability which is a major drawback for such a sensitive and crucial test.

\section{Conclusion}

We concluded from our study that both Quantus FLM and fetal pulmonary artery AT/ET are comparable in predicting fetal lung maturity and hence development of neonatal RDS. The advantages of quantus FLM were the ease to obtain the required ultrasound images and the reproducibility of the results which eliminates the interobserver variations. 


\section{List of Abbreviation}

RDS: neonatal respiratory distress syndrome

Quantus FLM: quantitative ultrasound fetal lung maturity

AT/ET: acceleration time/ejection time

MPA: main pulmonary artery

PSV: Peak systolic velocity

EDV: End diastolic velocity

PI: Pulsatility index

RI: Resistive index

$\mathrm{S} / \mathrm{D}$ : systolic diastolic ratio

\section{Study Design}

Observational prospective cross sectional study

\section{Ethics Declaration}

\section{Ethics Approval and Consent to Participate}

This study approved by ethics committee of the faculty of medicine of our university and consent to participate was taken

\section{Consent for Publication}

A written consent for publications was taken from all patients.

\section{Availability of Data and Material}

All data and material were available for this study

\section{Competing Interests}

The authors declare that they have no competing interests

\section{Funding:}

No funding received for this work from any organization

\section{Authors' Contributions}

All authors read and approved the final manuscript.

All authors made a significant contribution to the manuscript. NMO, SE and MK conceptualized the study. OAW and HSA were involved in the primary research concept and design of the study, initial data collection, writing, and data analysis. All authors participated in the study design, planning of analysis, and interpretation of results. -Acknowledgment; first of all to my god I'm gratefully indebted; nothings can be done without.

\section{References}

[1] Blencowe H, Cousens S, Oestergaard MZ, Chou D, Moller AB, Narwal R, Adler A, Garcia CV, Rohde S, Say L: National, regional, and worldwide estimates of preterm birth rates in the year 2010 with time trends since 1990 for selected countries: a systematic analysis and implications. The Lancet 2012, 379 (9832): 2162-2172.
[2] Maeda K, Serizawa M, Yamamoto N: Ultrasound tissue characterization with the gray level histogram width of the Bmode. The Ultrasound Review of Obstetrics \& Gynecology 2005, 5 (2): 92-95.

[3] Agrons GA, Courtney SE, Stocker JT, Markowitz RI: Lung disease in premature neonates: radiologic-pathologic correlation. Radiographics 2005, 25 (4): 1047-1073.

[4] Christian L, Hermansen M, Lorah M, Kevin N: Lancaster General Hospital, Lancaster, Pennsylvania Am Fam Physician. Oct 1 2007, 76 (7 SRC - BaiduScholar): 987-994.

[5] Azpurua H, Norwitz ER, Campbell KH, J.: Acceleration/ejection time ratio in the fetal pulmonary artery predicts fetal lung maturity. Am Gynecol 402010, 203 SRC BaiduScholar: e1-8.

[6] Osada H, Iitsuka Y, Masuda K, Sakamoto R, Kaku K, Seki K, Sekiya S: Application of lung volume measurement by threedimensional ultrasonography for clinical assessment of fetal lung development. Journal of ultrasound in medicine: official journal of the American Institute of Ultrasound in Medicine 2002, 21 (8): 841-847.

[7] Deter RL, Hadlock FP, Carollo BR: Ultrasonically determined menstrual age as an indicator of fetal lung maturity. Journal of clinical ultrasound: JCU 1982, 10 (4): 153-157.

[8] Harman CR, Manning FA, Stearns E, Morrison I: The correlation of ultrasonic placental grading and fetal pulmonary maturation in five hundred sixty-three pregnancies. American journal of obstetrics and gynecology 1982, 143 (8): 941-943.

[9] Tabsh KM: Correlation of ultrasonic epiphyseal centers and the lecithin: sphingomyelin ratio. Obstetrics and gynecology 1984, 64 (1): 92-96.

[10] Bonet-Carne E, Palacio M, Cobo T, Perez-Moreno A, Lopez M, Piraquive JP, Ramirez JC, Botet F, Marques F, Gratacos E: Quantitative ultrasound texture analysis of fetal lungs to predict neonatal respiratory morbidity. Ultrasound in Obstetrics \& Gynecology 2015, 45 (4): 427-433.

[11] Palacio M, Bonet-Carne E, Cobo T, Perez-Moreno A, Sabrià J, Richter J, Kacerovsky M, Jacobsson B, García-Posada RA, Bugatto F: Prediction of neonatal respiratory morbidity by quantitative ultrasound lung textureanalysis: a multicenter study. American journal of obstetrics and gynecology 2017, 217 (2): 196. e191-196. e114.

[12] Pramanik AK, Rangaswamy N, Gates T: Neonatal Respiratory Distress. Pediatric Clinics of North America 2015, 62 (2): 453-469.

[13] Moety GAFA, Gaafar HM, El Rifai NM: Can fetal pulmonary artery Doppler indices predict neonatal respiratory distress syndrome? Journal of Perinatology 2015, 35 (12): 1015-1019.

[14] Kim SM, Park JS, Norwitz ER, J.: Acceleration time-toejection time ratio in fetal pulmonary artery predicts the development of neonatal respiratory distress syndrome: a prospective cohort study. Am 805 2013, 30 SRC BaiduScholar.

[15] Kim S, Norwitz E, Hwang E, Kang H, Park C-W, Jun J, Park J Acceleration Time-to-Ejection Time Ratio in Fetal Pulmonary Artery Predicts the Development of Neonatal Respiratory Distress Syndrome: A Prospective Cohort Study. American Journal of Perinatology 2013, 30 (10): 805-812. 
[16] Ismael M: Study of the correlation of fetal main pulmonary artery Doppler indices and fetal lung maturity. Minia University; 2018.

[17] Guan Y, Li S, Luo G, Wang C, Norwitz ER, Fu Q, Tu X, Tian $\mathrm{X}, \mathrm{Zhu} \mathrm{J}$ : The role of doppler waveforms in the fetal main pulmonary artery in the prediction of neonatal respiratory distress syndrome. Journal of Clinical Ultrasound 2014, 43 (6): 375-383.

[18] Khanipouyani F, Abbasalizade F, Abbasalizade S, Fardiazar Z, Ghaffari S, Sarbakhshi P: PREDICTING FETAL LUNG MATURITY USING THE FETAL MAIN PULMONARY ARTERY DOP-PLER INDICES. Acta Medica Mediterranea 2016, 32 (Specia): 921-926.

[19] Büke B, Destegül E, Akkaya H, Şimşek D, Kazandi M: Prediction of neonatal respiratory distress syndrome via pulmonary artery Doppler examination. The Journal of Maternal-Fetal \& Neonatal Medicine 2017: 1-6.
[20] Schenone MH, Samson JE, Jenkins L, Suhag A, Mari G: Predicting Fetal Lung Maturity Using the Fetal Pulmonary Artery Doppler Wave Acceleration/Ejection Time Ratio. Fetal Diagnosis and Therapy 2014, 36 (3): 208-214.

[21] Chaoui R, Taddei F, Rizzo G: Doppler echocardiography of the main stems of the pulmonary arteries in the normal human fetus. Ultrasound Obstet Gynecol 173 1998, 11 SRC BaiduScholar.

[22] Mohamed MABE, EL-Didy HMA, Hosni AN, Gaafar HM, Alanwary SM: Acceleration/ejection time ratio in the fetal pulmonary artery predicts fetal lung maturity in diabetic pregnancies. 2015.

[23] Townsel CD, Emmer SF, Campbell WA, Hussain N: Gender Differences in Respiratory Morbidity and Mortality of Preterm Neonates. Frontiers in pediatrics 2017, 5: 6-6. 